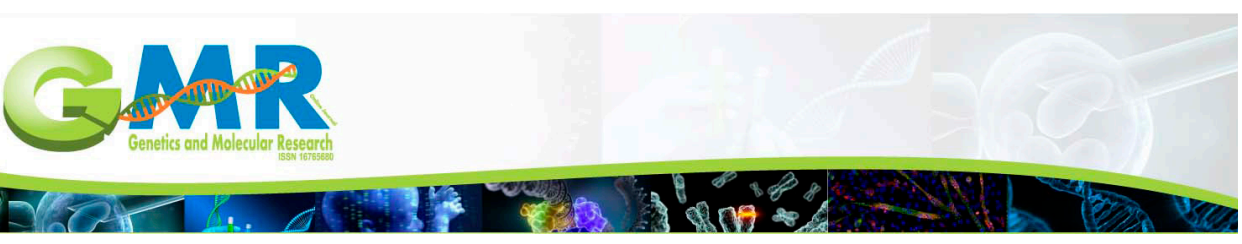

\title{
Genetic diversity in mutated and non-mutated rice varieties
}

\author{
M. Naeem ${ }^{1 *}$, F. Ghouri2*, M.Q. Shahid ${ }^{2 *}$, M. Iqbal', F. S. Baloch ${ }^{3}$, L. Chen ${ }^{2}$, \\ S. Allah', M. Babar ${ }^{4}$ and M. Rana ${ }^{1}$ \\ ${ }^{1}$ Department of Plant Breeding and Genetics, \\ University College of Agriculture and Environmental Sciences, \\ The Islamia University of Bahawalpur, Bahawalpur, Pakistan \\ ${ }^{2}$ College of Agriculture, South China Agricultural University, Guangzhou China \\ ${ }^{3}$ Department of Field Crops, Faculty of Agricultural and Natural Science, \\ Abant İzzet Baysal University, Bolu, Turkey \\ ${ }^{4}$ Institute of Biotechnology, Bahauddin Zakariya University, Multan, Pakistan \\ *These authors contributed equally to this study. \\ Corresponding authors: M.Q. Shahid / M. Naeem \\ E-mail: shahidmq@yahoo.com / mniub@iub.edu.pk \\ Genet. Mol. Res. 14 (4): 17109-17123 (2015) \\ Received August 17, 2015 \\ Accepted October 23, 2015 \\ Published December 16, 2015 \\ DOI http://dx.doi.org/10.4238/2015.December.16.11
}

\begin{abstract}
We studied the genetic variability due to mutation induced by $\mathrm{Y}$-rays $(10,15$, and $20 \mathrm{Kr})$ on various traits of twelve rice genotypes. Mutated and non-mutated seeds were sown in the field between July 2013 and 2014 using a split plot design. Yield and yield-related trait data was recorded, which showed significant $(P<0.05)$ genotypic and irradiation effects. Gamma radiation exerted non-significant effects on the panicle length of all plants, indicating the uniformity of performance of this character at different radiation levels. The plant height, grains per main panicle, panicle length, 1000-grain weight, grain weight per main panicle, and fertility percentage was minimum in Basmati-198, Basmati-Pak, Shaheen-2000, Super Basmati, Basmati-385, and Super Basmati, respectively, when exposed to radiation at $20 \mathrm{Kr}$. However, Basmati-370 attained maximum flag leaf area at this level of radiation. Broad sense heritability ranged from 72.0 to $97.7 \%$, indicating the
\end{abstract}


possibility of selection during earlier generations. Phenotypic correlation was positive and significant between grain weight per main panicle with panicle length and flag leaf area. Number of grains per main panicle and genotypic correlation were positive among grain weight per main panicle, panicle length, fertility percentage, 1000-grain weight, and plant height, indicating the effectiveness of these traits in the selection for yield. Phylogenetic analyses revealed a pair-wise similarity ranging from $0.51-0.76$ before mutation and $0.39-0.89$ after mutation. This study proved that $\mathrm{y}$-rays at $20 \mathrm{Kr}$ would be most effective in creating genetic variability in the existing germplasm.

Key words: Genetic diversity; Genetic variation; Mutagenesis; Mutation breeding; Oryza sativa

\section{INTRODUCTION}

Rice is an important cereal crop in Pakistan, an excellent source of foreign exchange earnings, and is the staple food of approximately half the world population. According to The Food and Agriculture Organization of the United Nations, 70.0\% more food must be produced in 2050 in order to feed a population of over nine billion people. Pakistan produces Basmati rice, which occupies a unique position in the world economy. Basmati rice accounts for $40.0 \%$, while coarse rice accounts for $60 \%$ of the national rice production. Rice crop contributes to $0.7 \%$ of the gross domestic product (GDP) of Pakistan and $3.1 \%$ of the value addition in agriculture. The aim of plant breeding is to improve crop production, as well as to improve the quality by changing the hereditary characteristics of plants. Semi-dwarf growth and rapid growth are important characteristics of rice breeding. There is an urgent need to adopt some innovative technologies to break the yield gap in rice, and to assist conventional breeding programs for the development of good varieties of rice. Greater knowledge of the genetic architecture of various genotypes is therefore necessary to formulate an efficient breeding methodology. lonizing radiations are an efficient source for the generation and improvement of genetic variability in rice crop. Historically, the use of mutagenesis in breeding has involved forward genetic screens, the selection of individual mutants with improved traits, and their incorporation into breeding programs. Inducible mutation is a suitable source of variations for crop improvement.

Exposure of plant material to ionic radiations inhibits meiosis. Among the different ionic radiations, gamma rays are frequently utilized in the crop improvement program, owing to their shorter wavelength, excessive energy per photon, and ability to penetrate deep within the tissues. However, the ability of these rays to induce genetic changes varies at an inter- and intra-specific level. The dimmers altering the strand finally alter normal base pairing, which hinders normal replication and transcription. Germination and survival decreases with the increase in radiation dosage of gamma rays and fast neutrons (Din et al., 2004). Gamma rays have been used to develop early maturing semi-dwarf varieties of rice. Increased intensity of gamma rays up to $35 \mathrm{Kr}$ remarkably reduces the number of tillers per plant, plant height, and length of main panicle. Ibrahim (2013) discovered that maximum variations in fertility percentage and filled grains per panicle could be achieved by deputing $25 \mathrm{Kr}$ gamma rays to Egyptian rice cultivar Sakha 105.

The mutation-based variety with higher yield potential YuaFengZao, was developed from IR8 using gamma radiations. The Nuclear Institute of Agriculture and Biology in Pakistan has 
developed 24 mutation-based varieties of various crops. As many as 3000 mutant varieties of crop plants (Shu and Lagoda, 2007) have been developed worldwide since the discovery of the mutation-inducing ability of X-rays in Drosophila melanogaster and barley. China has produced the largest number of mutation-based varieties of rice, ahead of India (11.5\%), the USSR and Russia (9.3\%), the Netherlands (7.8\%), USA (5.7\%), and Japan 5.3\%) (Ahloowalia et al., 2004). Of the 434 mutant varieties of rice, $225(56.0 \%)$ have been induced by gamma rays, and a majority $(67.0 \%)$ were developed as direct mutants (Maluszynski et al., 2001).

Repeated utilization of limited basmati varieties for the evolution of new varieties has narrowed the genetic base of recently evolved varieties in Pakistan. The development of broad gene-based varieties could efficiently slow down the evolution of strains and biotypes of parasites. Basmati 370 is the principal component of 4 major existing varieties of rice in Pakistan (Khan, 1996; Rabbani et al., 2008). Owing to the narrow genetic base, all aromatic varieties are susceptible to various biotic and abiotic stresses (Junaid et al., 2000). Genetic diversity can be estimated on the basis of morphological data; however, accuracy can be ensured by the use of DNA markers such as RAPD (Rabbani et al., 2008) and SSR markers (Shah et al., 2013). SSR markers have been used in a number of mutation analyses (Li et al., 2010), and correlation and genetic diversity studies (Zhao et al., 2010), owing to their easy availability throughout the genome.

\section{MATERIAL AND METHODS}

\section{Field studies}

Well filled, dry, healthy, dormant (200 g) seeds of Basmati-370, Basmati-Pak, Basmati-198, Basmati-385, Super Basmati, Basmati-2000, Shaheen-2000, Basmati-515, IRRI-6, KS-282, NiabIRRI-9, and KSK-133, with an average moisture content of $13.0 \%$ were treated in the gamma chamber at 10, 15, and $20 \mathrm{~kg}$ Roentgen for $30 \mathrm{~min}$ to 2 hours, depending on the dose of gamma radiation. The wave length of gamma rays ranged from 0.15 to $0.005 \AA$. The gamma irradiation was performed at the Nuclear Institute of Agriculture and Biology (NIAB), Faisalabad, Pakistan.

Treated seeds $\left(100 \mathrm{M}_{0}\right)$ of each genotype were cultivated in fully saturated loamy soil on

May, 2011, in order to obtain $M_{1}$ seeds. A thin layer of silt and farmyard manure mixture (3:1) was used to cover the seeds. The beds were moistened with showers of canal water twice a day. Wheat straw was used to save seedlings from heat shocks and conserve moisture, until the seedlings attained a height of $5 \mathrm{~cm}$. Thirty-day-old seedlings were transplanted to the field for space planting. All material was propagated for $\mathrm{M}_{1}$ in 2012 in the Experimental area of the Department of Plant Breeding and Genetics, UCA \& ES, The Islamia University of Bahawalpur. This evaluation was conducted in the $\mathrm{M}_{2}$ generation, grown in 2013 and 2014 at the same experimental area: $M_{2}$ seeds of each genotype subjected to each type of irradiation were divided into two parts; half was sown each year. The seeds were cultivated in a split plot design, with the original genotype cultivated in the main plots, and the gamma-irradiated seeds grown in the sub-plots (four replications). The sub-plot size was maintained by ensuring a row to row and plant to plant distance of $45 \mathrm{~cm}$ and 30 $\mathrm{cm}$, respectively. Standard agronomic and crop management practices were employed to maintain a healthy crop stand. Plant height, flag leaf area, panicle length, number of grains per panicle, grain weight per main panicle, fertility percentage, and seed index data was collected and analyzed. Data collected for each trait over two years was pooled before being analyzed. The genotypic and phenotypic correlation coefficients of twelve varieties were analyzed according to the formula given 
by Kwon and Torrie (1964). Data obtained over two years was pooled, as the yearly effect was not significant. Statistical analyses were performed using Fisher's analysis of variance (ANOVA), and the mean values were compared using the least difference test at a probability level of $5 \%$ (Steel et al., 1997) using Statistix 8.1 (Analytical Software, Tallahassee, FL, USA).

\section{Genetic diversity studies}

\section{Marker analysis}

During 2014, leaves were collected from young seedlings 15 days after transplantation, and DNA was extracted using a previously-described method (Liu et al., 2011). Eighty seven SSR primers equally distributed on twelve chromosomes (McCouch et al., 2002) were analyzed. The SSR analysis was done as described by Tong et al. (2011) and Wu et al. (2013). The amplified fragments were electrophoresed on a $1.2 \%$ agarose gel at $80 \mathrm{~V}$ in $1 \mathrm{X}$ TBE buffer (Yang et al., 2012). The gels were stained with ethidium bromide, and visualized in a gel documentation system.

\section{Marker data analysis}

Amplified products of each SSR primer were scored as present or absent $(1,0)$. The PowerMarker program (Liu and Muse, 2005) was used to calculate the number of alleles per locus for SSR analysis. Genetic similarity estimates were obtained for all pairs of cultivars according to the method described by Nei and Li (1979). The resulting similarity data was used to prepare a dendrogram, based on un-weighted pair group method with arithmetic average (UPGMA), to determine the genetic relationship among varieties. The phylogenetic tree was visualized using the TreeView software (Page, 2001).

\section{RESULTS AND DISCUSSION}

ANOVA revealed highly significant $(P<0.01)$ genotypic irradiation and the effects of its interaction on all traits of rice excluding the fertility percentage; only significant differences $(P<$ 0.05 ) were observed among the fertility percentages of all genotypes (Table 1).

\begin{tabular}{|c|c|c|c|c|c|c|c|c|}
\hline So. Var. & d.f & $\mathrm{PH}$ & FLA & $\mathrm{PL}$ & NGP & GWMP & FP & $\mathrm{SI}$ \\
\hline Replication & 2 & $54.2^{\mathrm{NS}}$ & $14.44^{\mathrm{NS}}$ & $1.58^{\mathrm{NS}}$ & $793.02^{\mathrm{NS}}$ & $0.16386^{\mathrm{NS}}$ & $9.819^{\mathrm{NS}}$ & $7.842^{\mathrm{NS}}$ \\
\hline Varieties & 11 & $1366.3^{* *}$ & $189.14^{* *}$ & $13.65^{\star \star}$ & $3797.13^{* *}$ & $2.93412^{* *}$ & $395.106^{*}$ & $23.004^{\star *}$ \\
\hline Error $_{1}$ & 22 & 30.6 & 9.34 & 0.82 & 335.93 & 0.09187 & 26.933 & 6.419 \\
\hline Radiation & 3 & $58098^{\star \star}$ & $1136.67^{\star \star}$ & $2656.67^{\star \star}$ & $5498.89^{\star *}$ & $0.37003^{\star *}$ & $108.640^{*}$ & $220.021^{\text {** }}$ \\
\hline$V^{*} R$ & 33 & $152.7^{\star \star}$ & $44.71^{\star \star}$ & $32.26^{* \star}$ & $815.00^{* *}$ & $0.29122^{\star *}$ & $233.795^{*}$ & $64.677^{\text {** }}$ \\
\hline $\begin{array}{l}\text { Error }_{2} \\
\text { Total }\end{array}$ & $\begin{array}{r}72 \\
133\end{array}$ & 36.9 & 11.17 & 1.36 & 375.65 & 0.11929 & 37.196 & 11.506 \\
\hline
\end{tabular}

NSNon significant. *, ${ }^{*}$ Significant at 5\%, 1\%. PH = plant height; FLA = flag leaf area; PL = panicle length; NGP = number of grains per panicle; GWMP = grain weight per main panicle; FP = fertility percentage; $\mathrm{SI}=$ seed index.

The interaction between genotypes and irradiation was significant; therefore, the means of both factors were compared separately. Mutated plants displayed obvious phenotypic changes, in the color, shape, and size, which distinguished them from non-irradiated plants, as previously 
reported by Haris et al. (2013). The post-hoc test revealed that all genotypes showed significant differences between their control and irradiated plants; however, within a single or among different doses of radiation, a majority of the genotypes showed non-significant differences (Tables 2-8).

Table 2. Comparison of mean values for Plant height of rice varieties at different radiation doses.

\begin{tabular}{|c|c|c|c|c|c|}
\hline Varieties & Control & $10 \mathrm{Kr}$ & $15 \mathrm{Kr}$ & $20 \mathrm{Kr}$ & Mean value \\
\hline Basmati-370 & $158.31^{1 \mathrm{~A}}$ & $59.53^{\mathrm{ac}}$ & $65.30^{\mathrm{aB}}$ & $60.80^{\mathrm{aC}}$ & 85.98 \\
\hline Basmati-Pak & $141.60^{\mathrm{bA}}$ & $52.00^{\mathrm{bB}}$ & $49.97^{\mathrm{dB}}$ & $51.07^{\mathrm{CB}}$ & 73.66 \\
\hline Basmati-198 & $127.95^{\mathrm{cA}}$ & $38.07^{\mathrm{eB}}$ & $37.73^{\operatorname{tgB}}$ & $37.40^{\mathrm{e} A \mathrm{~B}}$ & 60.28 \\
\hline Basmati-385 & $120.77^{\mathrm{cdA}}$ & $32.47 \mathrm{gr}$ & $33.93^{98}$ & $30.63^{98}$ & 54.45 \\
\hline Super Basmati & $115.80^{\mathrm{dA}}$ & $39.00^{\mathrm{eC}}$ & $43.10^{\mathrm{efB}}$ & $43.10^{d B}$ & 60.25 \\
\hline Basmati-2000 & $126.09^{c A}$ & $48.00^{\mathrm{bcB}}$ & $47.20^{\mathrm{deB}}$ & $51.33^{\text {bс }}$ & 68.15 \\
\hline Shaheen 2000 & $115.92^{\mathrm{dA}}$ & $47.13^{\mathrm{cB}}$ & $43.80^{\mathrm{efB}}$ & $47.30^{\mathrm{CdB}}$ & 63.53 \\
\hline Basmati-515 & $128.62^{\mathrm{CA}}$ & $46.47^{\mathrm{cB}}$ & $45.88^{\mathrm{eB}}$ & $46 .^{\mathrm{B}}$ & 66.87 \\
\hline IRRI-6 & $105.80^{\mathrm{eA}}$ & $39.73^{\mathrm{eB}}$ & $35.60^{\mathrm{gBC}}$ & $34.45^{\text {tc }}$ & 53.89 \\
\hline KS-282 & $113.65^{\mathrm{deA}}$ & $34.27^{1 \mathrm{~B}}$ & $33.60^{\mathrm{gB}}$ & $33.07^{18}$ & 53.64 \\
\hline NIAB-IRRI-9 & $126.25^{\mathrm{cA}}$ & $30.27^{9 \mathrm{~B}}$ & $30.13^{\text {hB }}$ & $28.30^{9 \mathrm{~B}}$ & 53.73 \\
\hline KSK-133 & $105.80^{\mathrm{eA}}$ & $35.13^{\text {eis }}$ & $34.30^{\mathrm{gB}}$ & $34.53^{18}$ & 52.44 \\
\hline Mean value & 123.88 & 41.83 & 41.71 & 41.54 & \\
\hline
\end{tabular}

LSD value $=9.7580$. Mean values sharing common letters for a given trait do not differ significantly at $5 \%$ probability level. Capital and small letters shows similarity and differences with in a same variety (rows) and among varieties (Columns).

Table 3. Comparison of mean values for flag leaf area of rice varieties at different radiation doses.

\begin{tabular}{|c|c|c|c|c|c|}
\hline Varieties & Control & $10 \mathrm{kR}$ & $15 \mathrm{kR}$ & $20 \mathrm{kR}$ & Mean value \\
\hline Basmati-370 & $35.40^{\mathrm{aA}}$ & $24.68^{\mathrm{bcB}}$ & $23.60^{\mathrm{aB}}$ & $18.27^{\mathrm{bc}}$ & 22.18 \\
\hline Basmati-Pak & $31.22^{\mathrm{bA}}$ & $22.91^{\mathrm{cB}}$ & $19.15^{b c}$ & $18.86^{\mathrm{bC}}$ & 20.31 \\
\hline Basmati-198 & $32.78^{\mathrm{abA}}$ & $7.20^{f C}$ & $9.04^{\mathrm{eB}}$ & $7.55^{\mathrm{fC}}$ & 7.93 \\
\hline Basmati-385 & $29.28^{\mathrm{bcA}}$ & $8.28^{\mathrm{fB}}$ & $8.99^{\mathrm{fB}}$ & $5.06^{\mathrm{fC}}$ & 7.45 \\
\hline Super Basmati & $29.65^{\mathrm{bcA}}$ & $22.44^{\mathrm{CB}}$ & $19.35^{\mathrm{bc}}$ & $21.79^{a B C}$ & 21.19 \\
\hline Basmati-2000 & $29.37^{\mathrm{bcA}}$ & $28.31^{1 \mathrm{~A}}$ & $19.18^{\mathrm{bB}}$ & $19.32^{\mathrm{abB}}$ & 22.27 \\
\hline Shaheen 2000 & $25.46^{\mathrm{cdA}}$ & $23.82^{\mathrm{bcAB}}$ & $19.76^{\mathrm{bB}}$ & $17.41^{\mathrm{bcB}}$ & 20.34 \\
\hline Basmati-515 & $23.25^{\mathrm{dA}}$ & $22.47^{\mathrm{cA}}$ & $21.13^{\mathrm{ab} A B}$ & $19.82^{\mathrm{bB}}$ & 21.14 \\
\hline IRRI-6 & $22.41^{\text {deA }}$ & $22.25^{\mathrm{dcA}}$ & $20.19^{\mathrm{abAB}}$ & $18.34^{\mathrm{bB}}$ & 20.26 \\
\hline KS-282 & $28.7^{\mathrm{bcA}}$ & $17.13^{\mathrm{dB}}$ & $17.31^{\mathrm{cdB}}$ & $17.62^{\mathrm{bcB}}$ & 17.36 \\
\hline NIAB-IRRI 9 & $28.57^{\mathrm{bcA}}$ & $15.58^{\mathrm{deB}}$ & $14.85^{\mathrm{cdB}}$ & $14.24^{\mathrm{cdB}}$ & 14.89 \\
\hline KSK-133 & $24.92^{\mathrm{dA}}$ & $12.46^{\mathrm{eC}}$ & $14.00^{\mathrm{cdB}}$ & $13.0^{\mathrm{cdBC}}$ & 13.15 \\
\hline Mean value & 28.33 & 18.96 & 17.21 & 15.94 & \\
\hline
\end{tabular}

$\mathrm{LSD}=5.37437580$. Mean values sharing the common letters for a given trait do not differ significantly at $5 \%$ probability level. Capital and small letters shows similarity and differences with in a same variety (rows) and among varieties (Columns).

\section{Plant height}

Plant height is an important agro-morphological attribute of crop plants that is generally indicative of the relative growth and vigor of crop plants. The genotypes showed a wide range of variability in plant height, varying from $28.30 \mathrm{~cm}$ (NIAB-IRRI-9 @ $20 \mathrm{Kr}$ ) to $158.31 \mathrm{~cm}$ (Basmati-370 @ 0 radiation) (Table 2 ). The plant height was significantly reduced in irradiated plants; for example, NIAB-IRI-9 irradiated at $20 \mathrm{Kr}$ showed a $97.95 \mathrm{~cm}$ reduction in plant height compared to its control. The post-hoc test revealed significant differences between the mean of control and irradiated plant heights; however, the three irradiated means of plant height showed only non-significant differences. These results are supported by the results of Wattoo et al. (2013), who reported a gradual decrease in plant height with the increase in gamma radiation intensity (15-25 kR); the plant height of mutated plants was significantly lower than that of control plants. 
Table 4. Comparison of mean values for Panicle length of rice varieties at different radiation doses.

\begin{tabular}{|c|c|c|c|c|c|}
\hline Varieties & Control & $10 \mathrm{kR}$ & $15 \mathrm{kR}$ & $20 \mathrm{kR}$ & Mean value \\
\hline Basmati-370 & $29.87^{\mathrm{aA}}$ & $10.26^{\mathrm{abB}}$ & $11.03^{\mathrm{aB}}$ & $10.73^{\mathrm{abB}}$ & 10.67 \\
\hline Basmati-Pak & $27.57^{\mathrm{bA}}$ & $9.80^{\mathrm{bB}}$ & $10.30^{\mathrm{bB}}$ & $9.53^{\mathrm{bB}}$ & 9.877 \\
\hline Basmati-198 & $28.26^{\mathrm{abA}}$ & $10.20^{\mathrm{abB}}$ & $10.10^{\mathrm{bB}}$ & $10.30^{\mathrm{abB}}$ & 10.2 \\
\hline Basmati-385 & $26.53^{\mathrm{bA}}$ & $11.33^{\mathrm{aB}}$ & $10.16^{\mathrm{bB}}$ & $11.16^{\mathrm{aB}}$ & 10.88 \\
\hline Super Basmati & $27.60^{\mathrm{bA}}$ & $9.60^{\mathrm{bB}}$ & $10.33^{\mathrm{bB}}$ & $10.20^{\mathrm{bB}}$ & 10.04 \\
\hline Basmati-2000 & $27.82^{\mathrm{bA}}$ & $10.60^{\mathrm{abB}}$ & $10.60^{\mathrm{bB}}$ & $10.76^{\mathrm{abB}}$ & 10.65 \\
\hline Shaheen 2000 & $27.31^{\mathrm{bA}}$ & $11.50^{\mathrm{aB}}$ & $10.83^{\mathrm{bB}}$ & $9.80^{\mathrm{cB}}$ & 10.71 \\
\hline Basmati-515 & $27.08^{\mathrm{bA}}$ & $11.13^{\mathrm{aB}}$ & $11.40^{\mathrm{aB}}$ & $10.53^{\mathrm{abB}}$ & 11.02 \\
\hline IRRI-6 & $23.49^{\mathrm{cA}}$ & $9.66^{\mathrm{bB}}$ & $8.56^{\mathrm{cB}}$ & $8.86^{\mathrm{bcB}}$ & 9.03 \\
\hline KS-282 & $27.58^{\mathrm{bA}}$ & $9.06^{\mathrm{bB}}$ & $8.83^{\mathrm{cB}}$ & $8.63^{\mathrm{bcB}}$ & 8.84 \\
\hline NIAB-IRRI 9 & $27.78^{\mathrm{bA}}$ & $8.10^{\mathrm{cB}}$ & $8.83^{\mathrm{cB}}$ & $7.73^{\mathrm{cB}}$ & 8.22 \\
\hline KSK-133 & $23.66^{\mathrm{cA}}$ & $8.40^{\mathrm{cB}}$ & $8.10^{\mathrm{cB}}$ & $8.06^{\mathrm{cB}}$ & 8.18 \\
\hline Mean value & 27.04 & 9.97 & 9.92 & 9.69 & 10.67 \\
\hline
\end{tabular}

$L S D=1.8138$. Mean values sharing the common letters for a given trait do not differ significantly at $5 \%$ probability level. Capital and small letters shows similarity and differences with in a same variety (rows) and among varieties (Columns).

Table 5. Comparison of mean values for Number of grains per panicle of rice varieties at different radiation doses.

\begin{tabular}{|c|c|c|c|c|c|}
\hline Varieties & Control & $10 \mathrm{kR}$ & $15 \mathrm{kR}$ & $20 \mathrm{Kr}$ & Mean value \\
\hline Basmati-370 & $162.27^{\mathrm{aA}}$ & $126.63^{\text {cdlJ }}$ & $128.63^{d l}$ & $134.63^{\mathrm{abH}}$ & 138.04 \\
\hline Basmati-Pak & $110.67^{\mathrm{fA}}$ & $69.30^{\text {hilJ }}$ & $75.30^{\text {iHI }}$ & $78.83^{9 \mathrm{H}}$ & 88.26667 \\
\hline Basmati-198 & $125.73^{\mathrm{eA}}$ & $111.73^{\mathrm{dD}}$ & $90.47^{\mathrm{ghlJ}}$ & $110.57^{\mathrm{dDE}}$ & 108.9233 \\
\hline Basmati-385 & $160.60^{\mathrm{abA}}$ & $118.27^{\text {deJK }}$ & $158.27^{\mathrm{aAB}}$ & $129.93^{\mathrm{bH}}$ & 149.6 \\
\hline Super Basmati & $124.80^{\mathrm{aE}}$ & $106.60^{\mathrm{eEF}}$ & $119.17^{\mathrm{eB}}$ & $74.17^{\mathrm{ghOP}}$ & 106.0467 \\
\hline Basmati-2000 & $163.60^{\mathrm{aA}}$ & $142.87^{\mathrm{bE}}$ & $130.00^{\mathrm{cdH}}$ & $114.27^{\mathrm{cdKL}}$ & 135.9567 \\
\hline Shaheen 2000 & $156.63^{\mathrm{Ba}}$ & $119.07^{\text {deK }}$ & $122.80^{\text {deJK }}$ & $141.23^{\mathrm{aEF}}$ & 140.22 \\
\hline Basmati-515 & $143.33^{\mathrm{Ca}}$ & $119.23^{\mathrm{deG}}$ & $129.33^{\mathrm{dDE}}$ & $127.23^{\mathrm{bcE}}$ & 133.2967 \\
\hline IRRI-6 & $143.43^{\mathrm{CA}}$ & $131.60^{\mathrm{CD}}$ & $117.53^{\mathrm{eGH}}$ & $130.17^{\mathrm{bDE}}$ & 135.0667 \\
\hline KS-282 & $156.63^{\text {ва }}$ & $147.93^{\mathrm{abc}}$ & $130.03^{\mathrm{cdGH}}$ & $135.57^{\mathrm{abF}}$ & 144.8633 \\
\hline NIAB-IRRI 9 & $136.93^{\mathrm{Da}}$ & $130.67^{\mathrm{cdBC}}$ & $111.10^{\mathrm{efH}}$ & $126.93^{\mathrm{cDE}}$ & 124.9867 \\
\hline KSK-133 & $159.83^{\mathrm{abA}}$ & $152.67^{\mathrm{ac}}$ & $152.47^{\mathrm{abc}}$ & $100.73^{e O P}$ & 154.99 \\
\hline Mean value & 111.85 & 146.1 & 125.41 & 123.70 & \\
\hline
\end{tabular}

LSD $=31.415$. Mean values sharing the common letters for a given trait do not differ significantly at $5 \%$ probability level. Capital and small letters shows similarity and differences with in a same variety (rows) and among varieties (Columns).

Table 6. Comparison of mean values for Grains Weight per main Panicle $(\mathrm{g})$ of rice varieties at different radiation doses.

\begin{tabular}{|c|c|c|c|c|c|}
\hline Varieties & Control & $10 \mathrm{KR}$ & $15 \mathrm{KR}$ & $20 \mathrm{KR}$ & Mean value \\
\hline Basmati-370 & $1.91^{\mathrm{bcA}}$ & $1.51^{\mathrm{ddB}}$ & $1.73^{\mathrm{bcAB}}$ & $1.71^{\mathrm{bAB}}$ & 1.71 \\
\hline Basmati-Pak & $1.65^{\mathrm{cA}}$ & $1.41^{\mathrm{cd} A \mathrm{~B}}$ & $1.21^{\mathrm{CB}}$ & $1.23^{\mathrm{bcAB}}$ & 1.37 \\
\hline Basmati-198 & $2.15^{\mathrm{bcA}}$ & $1.53^{\mathrm{cBC}}$ & $1.78^{\mathrm{bcB}}$ & $1.61^{\mathrm{bcBC}}$ & 1.76 \\
\hline Basmati-385 & $1.94^{\mathrm{bcA}}$ & $0.97^{\mathrm{cdCD}}$ & $0.98^{\mathrm{cdCD}}$ & $1.22^{\mathrm{bcBC}}$ & 1.27 \\
\hline Super Basmati & $2.22^{\mathrm{bcA}}$ & $1.70^{\mathrm{bCAB}}$ & $1.98^{\mathrm{bAB}}$ & $1.18^{\mathrm{bcCD}}$ & 1.77 \\
\hline Basmati2000 & $2.57^{\mathrm{abA}}$ & $2.09^{\mathrm{bcBC}}$ & $2.08^{\mathrm{abBC}}$ & $2.40^{\mathrm{abAB}}$ & 2.28 \\
\hline Shaheen2000 & $2.10^{\mathrm{bcA}}$ & $1.57^{\mathrm{bcBC}}$ & $1.66^{\mathrm{bcBC}}$ & $1.80^{\mathrm{abAB}}$ & 1.78 \\
\hline Basmati-515 & $2.53^{\mathrm{dA}}$ & $2.05^{\mathrm{bcBC}}$ & $2.13^{\mathrm{abB}}$ & $2.04^{\mathrm{abBC}}$ & 2.23 \\
\hline IRRI-6 & $2.81^{\mathrm{abA}}$ & $2.52^{\mathrm{abB}}$ & $2.49^{\mathrm{abAB}}$ & $2.40^{\mathrm{abB}}$ & 2.55 \\
\hline KS-282 & $3.29^{\mathrm{aA}}$ & $3.14^{\mathrm{aAB}}$ & $2.79^{\mathrm{aBC}}$ & $2.54^{\mathrm{aBC}}$ & 2.94 \\
\hline NIAB-IRRI-9 & $2.72^{\mathrm{abA}}$ & $1.86^{\mathrm{bcCD}}$ & $2.31^{\mathrm{abB}}$ & $1.80^{\mathrm{abCD}}$ & 2.17 \\
\hline KSK-133 & $3.0^{\mathrm{abA}}$ & $2.17^{\mathrm{bcCD}}$ & $2.32^{\mathrm{abBC}}$ & $2.45^{\mathrm{abBC}}$ & 2.49 \\
\hline Mean value & 2.41 & 1.87 & 1.79 & 1.69 & \\
\hline
\end{tabular}

LSD value $=0.5503$. Mean values sharing the common letters for a given trait do not differ significantly at $5 \%$ probability level. Capital and small letters shows similarity and differences with in a same variety (rows) and among varieties (Columns).

Genetics and Molecular Research 14 (4): 17109-17123 (2015) ～CFUNPEC-RP www.funpecrp.com.br 
Table 7. Comparison of mean values for Fertility Percentage of rice varieties at different radiation doses.

\begin{tabular}{|c|c|c|c|c|c|}
\hline Varieties & Control & $10 \mathrm{kR}$ & $15 \mathrm{kR}$ & $20 \mathrm{kR}$ & Mean value \\
\hline Basmati-370 & $79.83^{\mathrm{bcA}}$ & $73.73^{\mathrm{bcBC}}$ & $78.53^{\mathrm{bcAB}}$ & $61.24^{\mathrm{dDE}}$ & 73.59 \\
\hline Basmati-Pak & $86.33^{\mathrm{bA}}$ & $71.29^{\mathrm{cdCD}}$ & $82.11^{\mathrm{bcB}}$ & $62.66^{\mathrm{cdDE}}$ & 79.91 \\
\hline Basmati-198 & $93.33^{\mathrm{aA}}$ & $76.67^{\mathrm{bCDE}}$ & $82.33^{\mathrm{bC}}$ & $75.33^{\mathrm{bDE}}$ & 81.77 \\
\hline Basmati-385 & $78.53^{\mathrm{CA}}$ & $69.26^{\mathrm{cdBC}}$ & $71.45^{\mathrm{cdBC}}$ & $68.46^{\mathrm{bcc}}$ & 69.73 \\
\hline Super Basmati & $83.43^{\mathrm{bcA}}$ & $74.56^{\mathrm{bcc}}$ & $76.07^{\mathrm{cBC}}$ & $48.10^{\mathrm{efH}}$ & 66.24 \\
\hline Basmati-2000 & $81.3^{\mathrm{bcA}}$ & $74.27^{\mathrm{bcBC}}$ & $80.76^{\text {bcAB }}$ & $73.0^{\mathrm{bcBC}}$ & 78.78 \\
\hline Shaheen 2000 & $87.10^{\mathrm{abA}}$ & $82.07^{\mathrm{bD}}$ & $85.30^{\mathrm{abAB}}$ & $75.53^{\mathrm{bCD}}$ & 80.96 \\
\hline Basmati-515 & $93.20^{\mathrm{Aa}}$ & $88.10^{\mathrm{aB}}$ & $91.36^{\mathrm{aAB}}$ & $74.96^{\mathrm{bcDE}}$ & 90.88 \\
\hline IRRI-6 & $92.96^{\mathrm{abA}}$ & $84.53^{\mathrm{abBC}}$ & $89.83^{\mathrm{ab} A \mathrm{~B}}$ & $83.80^{\mathrm{ac}}$ & 87.1 \\
\hline KS-282 & $88.13^{\mathrm{abA}}$ & $73.74^{\mathrm{bcD}}$ & $82.38^{\mathrm{bB}}$ & $70.76^{\mathrm{bCDE}}$ & 75.63 \\
\hline NIABIRRI9 & $91.93^{\mathrm{abA}}$ & $85.16^{\mathrm{abD}}$ & $87.33^{\mathrm{abAB}}$ & $68.39^{\mathrm{cEF}}$ & 80.29 \\
\hline KSK-133 & $80.86^{\mathrm{bcA}}$ & $76.82^{\mathrm{bcAB}}$ & $76.56^{\mathrm{cdAB}}$ & $61.34^{\text {Dde }}$ & 71.57 \\
\hline Mean value & 80.94 & 79.39 & 78.03 & 76.69 & 73.59 \\
\hline
\end{tabular}

LSD value $=9.6534$. Mean values sharing the common letters for a given trait do not differ significantly at $5 \%$ probability level. Capital and small letters shows similarity and differences with in a same variety (rows) and among varieties (Columns).

\begin{tabular}{|c|c|c|c|c|c|}
\hline Varieties & Control & $10 \mathrm{kR}$ & $15 \mathrm{Kr}$ & $20 \mathrm{kR}$ & Mean value \\
\hline Basmati-370 & $20.91^{\mathrm{bAB}}$ & $21.08^{\mathrm{aA}}$ & $13.19^{\mathrm{cBC}}$ & $8.84^{\mathrm{deCD}}$ & 14.37 \\
\hline Basmati-Pak & $24.90^{\mathrm{abA}}$ & $20.59^{\mathrm{abAB}}$ & $9.18^{\mathrm{cdD}}$ & $12.15^{\mathrm{dCD}}$ & 13.97 \\
\hline Basmati-198 & $19.09^{\mathrm{bcA}}$ & $13.86^{\mathrm{bcBC}}$ & $19.34^{\mathrm{abA}}$ & $16.35^{\mathrm{cAB}}$ & 16.53 \\
\hline Basmati-385 & $16.48^{\mathrm{cB}}$ & $19.17^{\mathrm{abBC}}$ & $20.52^{\mathrm{abA}}$ & $8.98^{\mathrm{deCD}}$ & 16.22 \\
\hline SuperBasmati & $18.47^{\mathrm{bcA}}$ & $17.51^{\mathrm{abAB}}$ & $11.83^{\mathrm{cdBC}}$ & $7.22^{\mathrm{eCD}}$ & 12.19 \\
\hline Basmati-2000 & $18.49^{\mathrm{bCAB}}$ & $18.63^{\mathrm{abA}}$ & $16.44^{\mathrm{bc} A \mathrm{~B}}$ & $13.04^{\mathrm{cdB}}$ & 16.03 \\
\hline Shaheen 2000 & $14.14^{\mathrm{cdCD}}$ & $14.96^{\mathrm{bcCD}}$ & $15.51^{\mathrm{bcCD}}$ & $27.99^{\mathrm{aA}}$ & 19.49 \\
\hline Basmati-515 & $17.32^{\mathrm{bcAB}}$ & $11.79^{\mathrm{cBC}}$ & $18.25^{\mathrm{bA}}$ & $16.10^{\mathrm{cd} A \mathrm{~B}}$ & 15.38 \\
\hline IRRI-6 & $20.20^{\mathrm{bcA}}$ & $17.43^{\mathrm{abAB}}$ & $19.90^{\mathrm{ab} A \mathrm{~B}}$ & $20.01^{\mathrm{bcA}}$ & 19.11 \\
\hline KS-282 & $20.20^{\text {bCAB }}$ & $13.15^{\mathrm{bcc}}$ & $23.22^{\mathrm{aA}}$ & $9.73^{\mathrm{deCD}}$ & 15.37 \\
\hline NIAB-IRRI 9 & $20.76^{\mathrm{bA}}$ & $16.58^{\mathrm{bAB}}$ & $12.72^{\mathrm{cdBC}}$ & $11.45^{\mathrm{dec}}$ & 13.587 \\
\hline KSK-133 & $26.43^{\mathrm{aA}}$ & $13.01^{\mathrm{bCCD}}$ & $13.26^{\mathrm{CCD}}$ & $14.20^{\mathrm{cdCD}}$ & 13.49 \\
\hline Mean value & 19.78 & 16.48 & 16.11 & 13.84 & \\
\hline
\end{tabular}

LSD value $=5.2400$. Capital and small letters shows similarity and differences with in a same variety (rows) and among varieties (Columns).

Genotypic and phenotypic coefficients of variability for plant height are presented in Table 9. The genotypic coefficient of variation measures the variability of any trait. The extent of environmental influence on a trait indicates the magnitude of differences between the genotypic and phenotypic coefficients of variation. Large differences reflect high environmental influence, while small differences reveal high genetic influence (Idris and Muhammad, 2013). Phenotypic coefficients of variability (34.5\%) were slightly higher than the genotypic coefficients (34.15\%; Table 9 ), which indicated that the variation is due to genetic content, along with considerable influence by the environment. Similar findings were reported by Idris and Muhammad (2013) and Rao et al. (2014), who reported a high phenotypic and low genotypic coefficient of variation for plant height. Very high broad sense heritability $97.7 \%$ was recorded for plant height by Mehla et al. (2000), allowing for selection during the early attempts at improving plant height. 
Table 9. Heritability, Genotypic, and Phenotypic coefficient of variation for various traits of rice.

\begin{tabular}{llllc}
\hline Sr. No & Traits & $\mathrm{H}^{2 \%}$ & G.C.V\% & P.C.V\% \\
\hline 1 & Plant height & 97.7 & 34.15 & 34.5 \\
2 & Flag leaf area & 95.0 & 38.47 & 39.46 \\
3 & Panicle length & 93.8 & 14.5 & 15.05 \\
4 & No. of grains per main panicle & 91.1 & 27.01 & 28.2 \\
5 & Grains weight per main panicle & 96.8 & 47.8 & 48.63 \\
6 & Fertility \%age & 93.1 & 14.07 & 14.57 \\
7 & 1000-grains wt. & 72.0 & 14.09 & 16.6 \\
\hline
\end{tabular}

$\mathrm{h}^{2}=$ heritability, G.C.V\% = genotypic coefficient of variation, P.C.V\% = phenotypic coefficient of variation.

\section{Flag leaf area}

Flag leaf area is an essential character reflecting the yield and yield-contributing traits. All varieties showed a wide range of variability in the flag leaf area [5.069 cm² (Basmati-385 @ $20 \mathrm{Kr}$ ) to $35.407 \mathrm{~cm}^{2}$ (Basmati-370 @ Control)]. Basmati-385 showed the greatest reduction in flag leaf area (of $24.22 \mathrm{~cm}^{2}$ ) in response to gamma radiation at $20 \mathrm{Kr}$, compared to its control. Alternately, gamma radiation had the least effect on the flag leaf area of Basmati-2000 $\left(1.06 \mathrm{~cm}^{2}\right.$ when exposed to $10 \mathrm{Kr}$; Table 3).

This data indicated that a higher dose of gamma radiation led to a reduction in flag leaf area, the mutation in various segment of chromosome. Similar results were obtained by (Shereen et al., 2009) who noted that the leaf area continuously increased from 3-47\% in plants exposed to 150 Gy gamma radiation, while the flag leaf area did not change despite an increase in radiation under saline conditions. Similar findings were reported by Burton (1952) who reported that access to the genotypic and phenotypic coefficient of variability, together with heritability estimates, could provide information about the extent of advance to be expected from selection.

Genotypic and phenotypic coefficients of variations of flag leaf area are presented in Table 9. Phenotypic coefficients of variability $(39.46 \%)$ were slightly higher than the genotypic coefficient of variability $(38.47 \%)$ of flag leaf area, indicating that the variation was a result of the genetic content, was well as the environment. Similar findings were reported by Dhanwani et al. (2013) who reported that maximum phenotypic and minimum genotypic coefficient of variation in flag leaf area indicated the presence of variation, however appreciable, as a result of environmental factors. The high broad sense heritability $(95.0 \%)$ obtained for this trait reflected the ease of selection in earlier generations. However (Ananadarao et al. (2011) and Dhanwani et al. (2013), showed findings that were inconsistent with those of this study: they stated that a high broad sense heritability determining the selection in earlier generation would improve the flag leaf area.

\section{Panicle length}

The panicle length plays a significant role in raising the yield per unit area of rice crop. All varieties showed a wide range of variability in the panicle length, ranging from $7.733 \mathrm{~cm}$ in NIABIRRI-9 irradiated at $20 \mathrm{Kr}$ to $29.87 \mathrm{~cm}$ in control Basmati-370. Radiation reduced the panicle length, thereby imparting a vigilant effect on KSK-133, reducing the PL up to $15.6 \mathrm{~cm}$ at $20 \mathrm{Kr}$ (Table 4).

The genotypic and phenotypic coefficients of variation of panicle length are presented in Table 9. The phenotypic coefficients of variability $(15.05 \%)$ were slightly higher than those of the genotype $(14.5 \%)$, which indicated that the variation was due to genetic content, with countable influence by the environment. Similar results were reported by Watto et al. (2013) and Rao et al. 
(2014), who reported that the panicle length showed high phenotypic and low genotypic coefficient of variation, indicating the presence of genetic variation in addition to noticeable impact by the environment, thereby allowing for its utilization in improving panicle length. High broad sense heritability (93.8\%) was recorded, as in a previous study (Anandrao et al., 2011), which indicated that selection during an early generation could help improve this trait.

\section{Number of grains per panicle}

The number of grains per main panicle influences the grain weight, and is an important trait influencing the yield and yield-contributing characters. The varieties showed a wide range of variability in number of grains per main panicle. The mean value of number of grains per main panicle varied from 69.30 (Basmati Pak at $10 \mathrm{Kr}$ ) to 163.60 (Basmati-2000, control). The maximum effect of radiation on the grains per panicle at $10 \mathrm{Kr}$ was observed in Basmati Pak, revealing a difference of 41.37 grains per panicle compared to its control. Basmati-385 was least affected by radiation, as the mean value reduced by only 2.33 grains per panicle in response to radiation (Table 5). Similarly, Ibrahim and El-Degwy (2013) observed a range of genetic variability upon the application of different doses of gamma radiation in the number of grains per main panicle. Additionally, Haris et al. (2013) reported that 200 Gy of radiation could produce variation through induced mutation. Similar results were observed by El-Keredy (1990), who reported a gradual decrease in number of grains per main panicle with the increase in radiation dose from $15 \mathrm{kR}$ to $25 \mathrm{kR}$. Wattoo et al. (2013) observed a decrease in the number of grains per panicle as a result of increase in gamma radiation from $15 \mathrm{kR}$ to $25 \mathrm{kR}$; additionally, the mutant showed lesser number of grains per panicle compared to the non-radiated plant.

The genotypic and phenotypic coefficients of variability in number of grains per main panicle are presented in Table 9. The phenotypic coefficients of variation $(28.2 \%)$ were slightly higher than the genotypic coefficients of variation $(27.01 \%)$ in number of grains per main panicle, indicating that the variation is due to genetic content, as well as the environment. Similar results were reported by Rao et al. (2014), who revealed that high phenotypic coefficient of variation and low genotypic coefficient of variation in number of grains per main panicle was indicative of the presence of genetic variation, as well as environmental influence. The high broad sense heritability $(91.2 \%)$ of the number of grains per panicle reflected the higher transmissibility described by Rao et al. (2014), who reported that the measurement of a maximal broad sense heritability during an earlier generation would affect the selection process.

\section{Grain weight per main panicle}

The grain weight per main panicle is the main characteristic of higher yield. We observed a range of variability for this trait. Mean values varied from $0.97 \mathrm{~g}$ (Basmati-385 @ $15 \mathrm{Kr}$ ) to 3.21 g (KS-282 @ control). The maximum mean value was observed in KS-282, followed by KS282 and KSK-133, whereas the minimum grains weight per main panicle was recorded in Basmati-385 at $10 \mathrm{Kr}$. Similar research was conducted by Ibrahim and El-Degwy (2013), who revealed that genetic variability increased with the increase in gamma radiation intensity of grains weight per main panicle (Table 6).

In this study, the various doses of gamma radiation imparted different effects on different rice varieties. Basmati-515, IRRI-6, KS-282, NIAB-IRRI-9, and KSK-133 displayed higher grain 
weight per main panicle at different doses of radiation, and showed maximum genetic variability, which is desirable for the selection of crop breeds in breeding programs.

Genotypic and phenotypic coefficients of variation in grain weight per main panicle are presented in Table 9. Phenotypic coefficients of variability $(48.63 \%)$ were higher than the genotypic coefficients (47.8\%) for grains weight per main panicle, indicating that the variation is due to genetic content, with minimum influence from the environment. Similar results are reported by Bhutta et al. (2005), who reported a much higher phenotypic than genotypic coefficient of variation in grain weight per main panicle, indicating the occurrence of genetic variation with minimal interference from the environment. A high broad sense heritability $(96.8 \%)$ of the grain weight per main panicle reflected the ease of selection during earlier generations. Similar findings were reported by Agrawal (2003), who stated that a high broad sense of heritability indicates selection during an earlier generation, speeding up the improvement in grain weight per main panicle.

\section{Fertility percentage}

Rice varieties used in this study showed a wide range of variability in fertility percentage [48.1 $\%$ (Super Basmati at $20 \mathrm{Kr}$ ) to $93.33 \%$ (Basmati-198 at control)]. Similar research was conducted by Ibrahim and El-Degwy (2013), who observed a significant difference in fertility percentage in mutant rice cultivars. They also reported that a higher dose of gamma radiation reduced fertility percentage consistently, as observed in this study. In this study, the fertility percentage was affected by a gradual increase in radiation level. The fertility percentage was less influenced at $10 \mathrm{Kr}$, while maximum variability was observed at $20 \mathrm{kR}$. Super Basmati was more affected at $20 \mathrm{kR}$ compared to the other varieties, which is an important achievement for further breeding programs (Table 7).

The genotypic and phenotypic coefficients of variation in fertility percentage are presented in Table 9. The phenotypic coefficients of variability $(14.57 \%)$ were non-significantly higher than that of the genotype $(14.07 \%)$ in fertility percentage, indicating that the variation is due to genetic content, with minimal involvement of the environment. Similar results were reported by Vanisree et al. (2013), who observed a slightly higher phenotypic than genotypic coefficient of variation in fertility percentage. The estimation of high broad sense heritability (93.1\%) in fertility percentage reflected the ease of selection during an earlier generation (Vanisree et al., 2013).

\section{0-grain weight}

The 1000 grain-weight is a major character influencing the yield and yield-contributing effect. The varieties showed a wide range of variability in 1000-grain weight at different radiation doses, varying from $7.22 \mathrm{~g}$ to $27.99 \mathrm{~g}$ (Table 8). The maximum mean 1000 -grain weight value was observed in Shaheen-2000 at $20 \mathrm{Kr}$, which was $13.85 \mathrm{gm}$ greater than its control. Similar results were reported by Bughio et al. (2007), who observed a remarkable mutagenic improvement in the 1000-grain weight of different rice varieties. Similar results were observed by Ibrahim and ElDegwy (2013), who reported a significant difference in the 1000-grain weight in various genotypes, when exposed to 15-25 kR radiation. A comparable result was obtained by Siddiqui and Singh (2010), who observed a higher mean value of 1000-grain weight at different radiation doses, which produced highly significant results among genotypes.

In this study, mutagenic treatments altered the 1000-grain weight in all varieties. Consequently, the mean 1000-grain weights of rice subjected to all types of treatment were virtually 
the same; moreover, the range of variability of irradiated and control plants were similar. Therefore, the gamma-ray dosages utilized in this study were capable of inducing a marked variation in the 1000-grain weight. Nevertheless, the differences among the 3 mutagenic treatments were significant.

The extent of environmental influence on this trait is depicted by the magnitude of differences between the genotypic and phenotypic coefficients of variation. Large differences reflect a high environmental influence, while small differences indicated a high genetic influence (Idris and Mohamed, 2013). The genotypic and phenotypic coefficients of variation in 1000-grain weight are presented in Table 9. The phenotypic coefficients of variability $(16.6 \%)$ are higher than the genotypic coefficients $(14.09 \%)$ of grains weight per main panicle, indicating that the variation is due to genetic effects (Rao et al., 2014). A moderate broad sense heritability $(72.0 \%)$ was recorded, similar to that in a previous study (Idris and Mohamed, 2013).

\section{Correlation analyses}

The crop yield is a very complex trait controlled by many genes and influenced by other traits. Therefore, other yield-related traits must be considered when selecting genotypes for yield. Correlation is an easy and efficient means to determine the association between different traits. In this study, phenotypic and genotypic correlation was evaluated. A majority of the traits depicted significant $(P<0.5)$ correlation among themselves, whereas the correlation among some traits was non-significant $(P>0.5)$. Maximum genotypic and phenotypic correlation was observed between the plant height and panicle length $(r g=0.7465, \mathrm{rp}=0.7294)$ followed by fertility percentage and 1000 -grain weight $(\mathrm{rg}=0.7355, \mathrm{rp}=0.5551)$. The flag leaf area and 1000-grain weight showed a non-significant correlation with a maximum number of traits, while the plant height and panicle length were significantly correlated with a maximum number of traits (Table 10). These results are supported by the findings of other researchers, who obtained similar results, when investigated the correlation among yield-related traits of rice (Shanthakumar et al., 1998; Ashfaq et al., 2012; Idris and Mohamed, 2013; Vanisree et al., 2013).

\begin{tabular}{|c|c|c|c|c|c|c|c|}
\hline Traits & $\mathrm{CR}$ & $\begin{array}{l}\text { Panicle length } \\
(\mathrm{cm})\end{array}$ & $\begin{array}{l}\text { No. of grains/ } \\
\text { panicle }\end{array}$ & $\begin{array}{l}\text { Grains weight/ } \\
\text { panicle }\end{array}$ & $\begin{array}{l}\text { Fertility } \\
\text { percentage }\end{array}$ & $\begin{array}{l}\text { Flag leaf area } \\
\qquad\left(\mathrm{cm}^{2}\right)\end{array}$ & $\begin{array}{c}1000 \text { grains } \\
\text { weight }(\mathrm{g})\end{array}$ \\
\hline \multirow[t]{2}{*}{ Plant height (cm) } & $\mathrm{rg}$ & $0.7465^{\star}$ & $-0.4241^{*}$ & $-0.4763^{*}$ & $-0.2657^{\star}$ & $-0.6551^{*}$ & $-0.2643^{*}$ \\
\hline & $\mathrm{rp}$ & $0.7294^{\star *}$ & $-0.4061^{*}$ & $-0.4623^{\star *}$ & $-0.2411^{\mathrm{NS}}$ & $-0.6395^{\star \star}$ & $-0.2333^{\mathrm{NS}}$ \\
\hline \multirow[t]{2}{*}{ Panicle length $(\mathrm{cm})$} & $\mathrm{rg}$ & & $-0.2188^{*}$ & $-0.6191^{*}$ & $-0.1458^{\star}$ & $0.3498^{*}$ & $-0.2942^{\mathrm{NS}}$ \\
\hline & $\mathrm{rp}$ & & $-0.2136^{\mathrm{NS}}$ & $-0.5986^{\star \star}$ & $-0.1561^{\mathrm{NS}}$ & $0.3200^{\text {NS }}$ & $-0.2648^{\mathrm{NS}}$ \\
\hline \multirow[t]{2}{*}{ No. of grains/ panicle } & $\mathrm{rg}$ & & & $0.5508^{\mathrm{NS}}$ & $0.1122^{*}$ & $-0.2702^{*}$ & $0.3474^{*}$ \\
\hline & $\mathrm{rp}$ & & & $0.5397^{\star \star}$ & $0.1453^{\mathrm{NS}}$ & $-0.2700^{\mathrm{NS}}$ & $0.2479^{\mathrm{NS}}$ \\
\hline \multirow[t]{2}{*}{ Grains weight/ panicle } & $\mathrm{rg}$ & & & & $0.4274^{*}$ & $0.0855^{\mathrm{NS}}$ & $0.3414^{\mathrm{NS}}$ \\
\hline & $\mathrm{rp}$ & & & & $0.4263^{* *}$ & $0.834^{\mathrm{NS}}$ & $0.2657^{\mathrm{NS}}$ \\
\hline \multirow[t]{2}{*}{ Fertility percentage } & $\mathrm{rg}$ & & & & & $-0.0176^{\mathrm{NS}}$ & $0.7355^{\star}$ \\
\hline & $\mathrm{rp}$ & & & & & $0.0042^{\mathrm{NS}}$ & $0.5551^{* \star}$ \\
\hline \multirow[t]{2}{*}{ Flage leaf area $\left(\mathrm{cm}^{2}\right)$} & $\mathrm{rg}$ & & & & & & $-0.1148^{\mathrm{NS}}$ \\
\hline & $\mathrm{rp}$ & & & & & & $-0.1347^{\mathrm{NS}}$ \\
\hline
\end{tabular}

NSNon-significance. ${ }^{*} \mathrm{P} \leq 0.05 \%$. CR = correlation, $\mathrm{rg}=$ genotypic correlation, $\mathrm{rp}=$ phenotypic correlation.

\section{Genetic diversity studies}

A phylogenetic analysis and similarity index of 12 coarse and fine varieties of rice (Basmati-370, Basmati Pak, Basmati-198, Basmati-515, Basmati-2000, Shaheen 2000, Basmati 
385, Super Basmati, KSK-282, KSK-133, IRRI-6, and NIAB-IRRI-9) before and after mutagenesis using 87 SSR markers (widely distributed on 12 chromosomes) were conducted. Genotypic banding patterns of 12 rice varieties with two SSR markers are shown in Figures 1 and 2 .

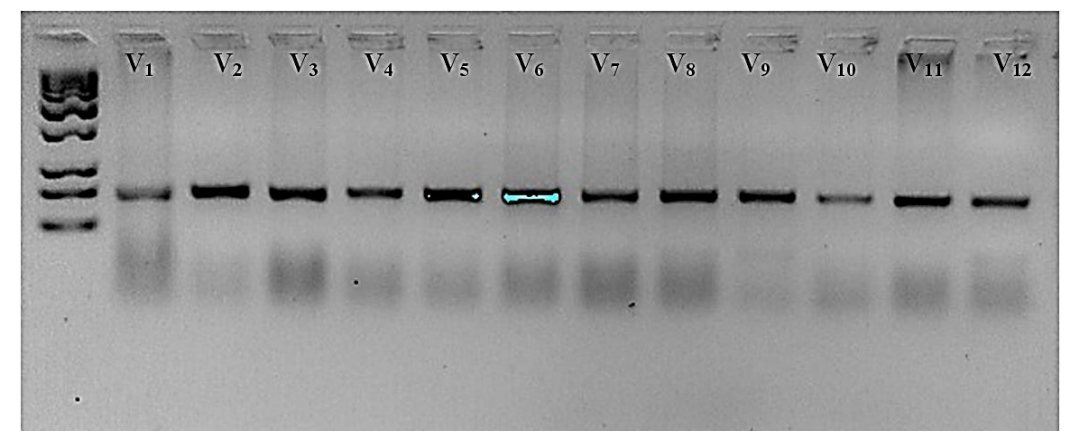

Figure 1. Gel electrophoresis of twelve rice varieties with SSR marker RM3202.

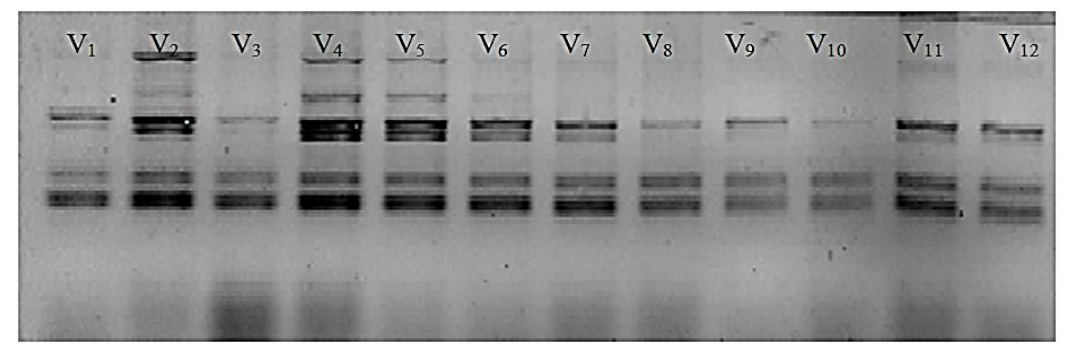

Figure 2. Gel electrophoresis of twelve rice varieties with SSR marker RM529.

Genetic analysis led to the grouping of rice varieties into two clades: fine (Basmati-370, Basmati Pak, Basmati-515, Basmati-2000, Shaheen 2000, Basmati 385, and Super Basmati) in clade-I, and coarse (KSK-282, KSK-133, IRRI-6, and NIAB-IRRI-9) in clade-II (Figure 3). A total of 153 polymorphic alleles were detected from 87 markers. A UPGMA-based dendrogram with two clusters was observed (Figure 3). Pairwise similarity ranged from 0.51 to 0.76 before (Figure 3 ) and 0.39 to 0.89 after mutagenesis (Figure 4 ).

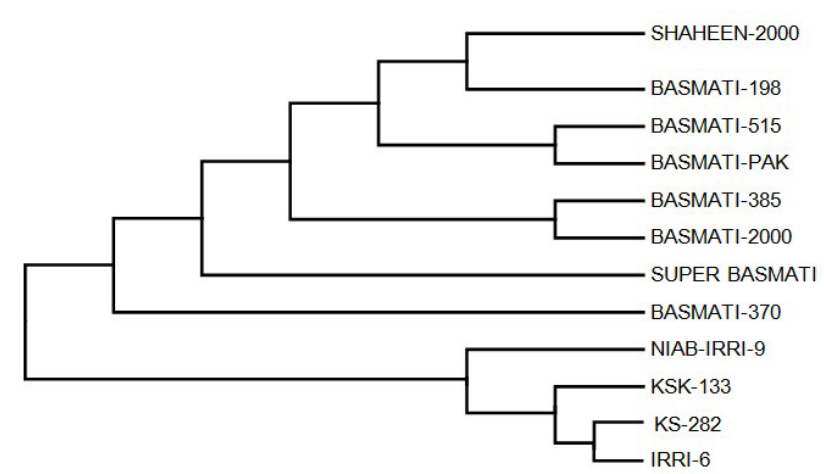

Figure 3. UPGMA cluster analysis showing the diversity and correlation among 12 rice varieties before mutagenesis. 


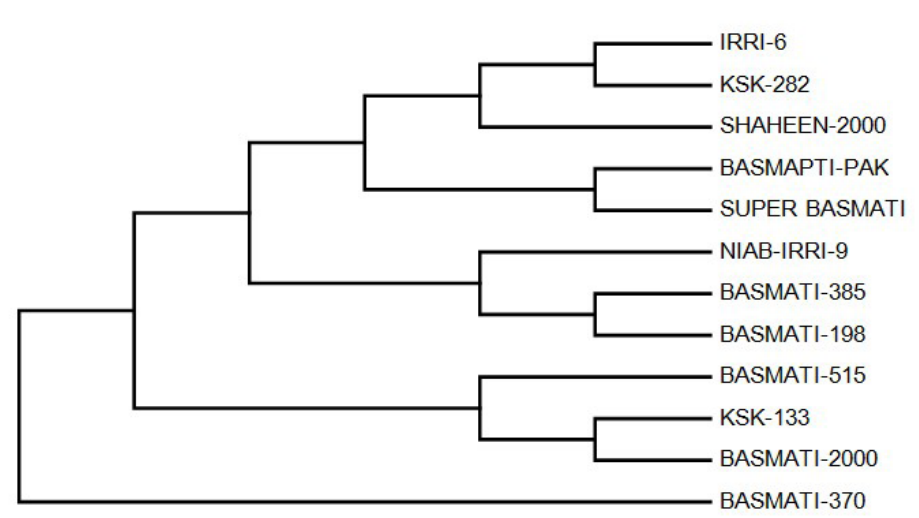

Figure 4. UPGMA cluster analysis showing the diversity and association between 12 rice varieties after mutagenesis.

Genetic distances between Basmati varieties were as low as 0.5-0.61 (Arif et al., 2005; Shah et al., 2013). A substantial genetic diversity was observed using SSR markers of various rice genotypes, and by clustering their results into 2, 3, 2, 3, and 4 groups. Similarly, the use of 87 SSR markers to determine the genetic diversity of mutated population revealed 187 polymorphic alleles in twelve varieties. Also, diversity in the genetic constitution was widened up to 0.39-0.89 after mutagenesis, and four clusters were developed by UPGMA. Our results are in accordance with those of Arif et al. (2005), who observed an increase in the diversity of mutated population compared to their respective controls. As a result of mutation (Figure 4), fine rice varieties Basmati-515 and Basmati 2000 were shuffled into the coarse variety cluster, while IRRI-6 and KSK-282 were incorporated into the fine variety group, indicating the effectiveness of gamma radiation for mutation. In conclusion, although the genetic base of Basmati varieties was diversified post-mutation, the varieties were less affected than non-Basmati varieties. Differences in the morphological uniqueness of Basmati and non-Basmati varieties remained unaltered even after mutagenesis. Twelve varieties with heading days 100-120 and plant height $60-110 \mathrm{~cm}$ showed a wide diversity among mutant and non-mutant lines. Genetic diversity generated by this mechanism can enrich the germplasm of rice crop, as the availability of a diverse gene pool for various economical traits can facilitate the crop improvement program.

\section{Conflicts of interest}

The authors declare no conflict of interest.

\section{ACKNOWLEDGMENTS}

Research supported by the Guangdong Provincial Key Natural Science Foundation (\#2014A030311042) and the Guangdong science and technology program (\#2014A030304055).

\section{REFERENCES}

Agrawal KB (2003). Variability studies in segregating populations of rice. Ann. Agric. Res. 24: 707-709.

Ahloowalia BS, Maluszynski M and Nichterlein K (2004). Global impact of mutation-derived varieties. Euphytica 135: $187-204$. 
Anandrao SD, Singh CM, Suresh BG and Lavanya GR (2011). Evaluation of rice hybrids for yield and yield component characters under North East Plain Zone. Allahabad Farmer 67: 63-68.

Arif M, Kousar S, Ajwa MA, Arif A, et al. (2005). Genetic diversity among rice genotypes of Pakistan through random amplified polymorphic DNA (RAPD) analysis. Pak. J. Bot. 37: 585-592.

Ashfaq M, Khan AS, Khan SHU and Ahmad RV (2012). Association of various morphological traits with yield and genetic divergence in rice (Oryza sativa L.). Int. J. Agric. Biol. 14: 55-62.

Bhutta WM, Barley T and Ibrahim M (2005). Path-coefficient analysis of some quantative characters in husked barley. Caderno de Pesquisa Ser Biologia Santa Cruz de Sul 17: 65-70.

Bughio HR, Dhano O, Asad MA and Bughio MS (2007). Improvement of grain yield in rice variety Basmati-370 (Oryza sativa L.), through mutagenesis. Pak. J. Bot. 39: 2463-2466.

Burton GW (1952). Quantitative Inheritance of Grasses. Proceedings of the 6th International Grassland Congress, Pennsylvania. 1: $277-283$.

Dhanwani RK, Sarawgi AK, Solanki A and Tiwari JK (2013). Genetic variability analysis for various yield attributing and quality traits in rice (Oryza sativa L.). Bioscan 8: 1403-1407.

Din R, Qasim M and Ahmad K (2004). Radio sensitivity of various wheat genotypes in meration. Int. J. Agric. Biol. 6: 898-900.

El-Keredy MS (1990). Induced useful mutations of some field crops in Egypt. Paper presented at the fourth Conference of Agronomy, University of Cairo, Egypt, 1: 237-253.

Haris A, Abdullah, Bakhtiar, Subaedah, et al. (2013). Gamma ray radiation mutant rice on local aged dwarf. Middle East J. Sci. Res. 15: 1160-1164.

Ibrahim S and El-Degwy (2013). Mutation induced genetic variability in rice (Oryza sativa L.). Int. J. Agric. Crop Sci. 5: 27892794.

Idris AE and Mohamed KA (2013). Estimation of genetic variability and correlation for grain yield components in rice (Oryza sativa L.). Glob. J. Plant Ecophysiol. 3: 1-6.

Idris AE, Justin FJ, Dagash YMI and Abuali Al (2012). Genetic variability and inter relationship between rice genotypes for yield and yield components. J. Plant Breed. Genet. 1: 73-84.

Junaid AK, Jamil FF and Gill MA (2000). Screening of rice varieties/lines against Bakanae and bacterial leaf blight. Pak. J. Phytopathol. 12: 6-11.

Khan MG (1996). A brief note on rice research institute Kala Shah Kaku. In: Report of the monitoring visit on fine grain aromatic rice in India, Iran, Pakistan and Thailand (Singh RK, Singh US and Khush GS, eds.). IRRI, Manila, 96-101.

Kwon SH and Torrie JH (1964). Heritability and interrelationship of traits of two soybean populations. Crop Sci. 4: 196-198.

Li W, Wu J, Weng S, Zhang D, et al. (2010). Characterization and fine mapping of the glabrous leaf and hull mutants (gl1) in rice (Oryza sativa L.). Plant Cell Rep. 29: 617-627.

Liu B, Li JQ, Liu XD, Shahid MQ, et al. (2011). Identification of neutral genes at pollen sterility loci Sd and Se of cultivated rice (Oryza sativa) with wild rice (O. rufipogon) origin. Genet. Mol. Res. 10: 3435-3445.

Liu KJ and Muse SV (2005). Power Marker an integrated analysis environment for genetic marker analysis. Bioinformatics 2: 2128-2129.

Maluszynski M, Nichterlein K, Zanten LV and Ahloowalia BS (2001). Officially released mutant varieties - the FAO/IAEA Database. Mut. Breed. Rev. 12: 1-84.

McCouch SR, Chen X and Cho YG (2002). Sequence divergence of rice microsatellite in Oryze and other species. Mol. Genet. Gen. 268: 331-334.

Mehla BS, Sharma SC and Hooda JS (2000). Gene action for certain quantitative traits in wheat. Annal. Biol. (Ludhiana). 16: 95-100.

Nei M and Li WH (1979). Mathematical model of studying genetic variation in terms of restriction endonucleases. Proc. Natl. Acad. Sci. U. S. A. 76: 5269-5276.

Panaud O, Chen X and McCouch SR (1996). Development of microsatellite markers and characterization of simple sequence length polymorphism (SSLP) in rice (Oryza sativa L.). Mol. Gen. Genet. 252: 597-607.

Rabbani MA, Pervaiz ZH and Masood MS (2008). Genetic diversity analysis of traditional and improved cultivars of Pakistan rice (Oryza sativa L.) using RAPD markers. Elec. J. Biotechnol. 11: 1-9.

Rao VT, Mohan YC, Bhadru D, Bharathi D, et al. (2014). Genetic variability and association analysis in rice. Inter. J. Appl. Sci. Pharmaceut. Technol. 5: 63-65.

Shah MS, Naveed SA and Arif M (2013). Genetic diversity in basmati and non-basmati rice varieties based on microsatellite markers. Pak. J. Bot. 45: 423-431.

Shanthakumar G, Mahadevappa M and Radraradhya M (1998). Variability, correlation and path analysis in rice (Oryza sativa L.) over seasons. Karnataka J. Agric. Sci. 11: 67-72.

Shereen Y, Ansari AR, Mumtaz S, Buhgio HR, et al. (2009). Impact of gamma irradiation induced changes and physiological 
responses of rice under saline conditions. Pak. J. Bot. 41: 2487-2495.

Shu QY and Lagoda PJL (2007). Mutation techniques for gene discovery and crop improvement. Mol. Plant Breed. 5: 193-195. Siddiqui SA and Sanjeeva S (2010). Induced genetic variability for yield and yield traits in basmati rice. World J. Agric. Sci. 6: 331-337.

Steel RGD, Torrie JH and Deekey DA (1997). Principles and procedures of statistics: A biometrical approach. 3rd edn. McGraw Hill Book Co. Inc., New York.

Tong JF, Li YH, Yang YX, Shahid MQ, et al. (2011). Molecular evolution of rice $S_{5}{ }^{n}$ and functional comparison among different sequences. Chinese Sci. Bull. 56: 2016-2024.

Vanisree S, Sawpna K, Raju ChD, Raju ChS, et al. (2013). Genetic variability and selection criteria in rice. J. Biol. Sci. Opin. 1: $341-346$.

Wattoo JI, Aslam K, Shah SM, Shabir G, et al. (2012). Ethyl methane sulphonate (EMS) induced mutagenic attempts to create genetic variability in Basmati rice. J. Plant Breed. Crop Sci. 4: 101-105.

Wu JW, Hu CY, Shahid MQ, Guo HB, et al. (2013). Analysis on genetic diversification and heterosis in autotetraploid rice. Springer Plus 2: 1-12.

Yang YX, Li YH, Tong JF, Shahid MQ, et al. (2012). Wide-compatibility gene $S_{5}{ }^{n}$ exploited by functional molecular markers and its effect on fertility of intersubspecific rice hybrids. Crop Sci. 52: 669-675.

Zhao K, Wright M, Kimball J, Eizenga G, et al. (2010). Genomic diversity and introgression in O. sativa reveal the impact of domestication and breeding on the rice genome. PLoS One 5: 10780. 